\title{
A Riesz-Haviland type result for truncated moment problems with solutions in $L^{1}$
}

\author{
C.-G. Ambrozie
}

\begin{abstract}
We give a version of the Riesz-Haviland theorem for truncated moments problems, characterizing the existence of the representing measures that are absolutely continuous with respect to the Lebesgue measure. The existence of such representing densities describes the dense interior of the convex cone of all data having nonnegative Borel representing measures. A natural regularity assumption on the support is required.

Keywords: positive functional, moments problem, measure MSC-class: 44A60 (Primary) 49J99 (Secondary)
\end{abstract}

\section{Introduction}

In this paper we consider problems of moments in $n$ real variables $t=\left(t_{1}, \ldots t_{n}\right)$, with respect to a finite number of monomial functions $t^{i}=t_{1}^{i_{1}} \cdots t_{n}^{i_{n}}$ where $i=\left(i_{1}, \ldots, i_{n}\right) \in\left(\mathbb{Z}_{+}\right)^{n}$, for Lebesgue integrable densities $f=f(t) \geq 0$ a.e. (almost everywhere) on closed subsets $T \subset \mathbb{R}^{n}$. Given a set of numbers $g_{i}$ with $|i|=i_{1}+\cdots+i_{n} \leq 2 k$ where $k \in \mathbb{N}$ is fixed, the truncated problem of moments, called also $T$-problem of moments [6] when $T$ is prescribed, is concerned with the existence of the Borel measures $\nu \geq 0$ on $\mathbb{R}^{n}$ supported on $T$ such that $\int_{T} t^{i} d \nu(t)=g_{i}$ for all $i$ [1], 18. One calls $\nu$ a representing measure for $g$, and $g_{i}$ the moments of $\nu$. We are interested in those representing measures $\nu=f d t$, called representing densities of $g$, that are absolutely continuous with respect to the Lebesgue measure $d t=d t_{1} \cdots d t_{n}$. For any subset $I \subset \mathbb{Z}_{+}^{n}$, let $P_{I}$ denote the linear span of the monomials $X^{i}(i \in I)$ in $\mathbb{R}\left[X_{1}, \ldots, X_{n}\right]$. Given $g=\left(g_{i}\right)_{i \in I}$, the linear Riesz functional $\varphi_{g}: P_{I} \rightarrow \mathbb{R}$ associated to $g$ is defined as it is known [7] by $\varphi_{g} X^{i}=g_{i}$ for $i \in I$. We say that $\varphi_{g}$ is $T$-positive [13] if $\varphi_{g} p \geq 0$ for all polynomials $p$ such that $p(t) \geq 0$ for all $t \in T$. This condition is necessary for $g$ to have representing measures $\nu$ on $T$, since in this case $\varphi_{g} p=\int_{T} p d \nu$ for all polynomials $p$. We remind below the Riesz-Haviland theorem [13, a basic result concerned with the full problem of moments when $I=\mathbb{Z}_{+}^{n}$.

Theorem 1 [13 Let $T \subset \mathbb{R}^{n}$ be closed, and $g=\left(g_{i}\right)_{i \in \mathbb{Z}_{+}^{n}}$ be a set of reals. Then $g$ has representing measures on $T$ if and only if $\varphi_{g}$ is $T$-positive.

An analogue of the Riesz-Haviland theorem for the truncated case was established by R.E. Curto and L.A. Fialkow [6]. For $I=I_{2 k}=\{i:|i| \leq 2 k\}$, 
when $P_{I}$ is the space of all polynomials of degree $\leq 2 k$, they proved that a set $g=\left(g_{i}\right)_{|i| \leq 2 k}$ has representing measures on $T$ if and only if the Riesz functional $\varphi_{g}: P_{I_{2 k}} \rightarrow \mathbb{R}$ admits $T$-positive extensions $\tilde{\varphi}_{g}: P_{I_{2 k+2}} \rightarrow \mathbb{R}$ to the space $P_{I_{2 k+2}}$ of all polynomials of degree $\leq 2 k+2$.

By Theorem 7, we characterize the existence of the representing densities of $g$ on regular supports $T$ by the condition: $\varphi_{g} p>0$ for all $p \in P_{I} \backslash\{0\}$ such that $p(t) \geq 0$ for all $t \in T$. A similar characterization [14] holds under more specific hypotheses, in particular if a distinguished moment $\tau_{i_{0}}\left(=t^{i_{0}}\right.$ or linear

combination of the $t^{i}$, s) exists such that $\lim _{\|t\| \rightarrow \infty} \frac{\left|t^{i}\right|}{1+\tau_{i_{0}}(t)}=0$ for $i \neq i_{0}$. We mention also the existence of similar results 11 for quadratic $(k=1)$ and some quartic $(k=2, n=2) T$-problems of moments.

By Theorem [6, the set of all $g$ having representing densities is the dense interior of the set of all $g$ having representing measures. Our proofs are independent of the results from [6], 14 and rely mainly on Theorem 1 [13].

Started by works of Stieltjes, Hausdorff, Hamburger and Riesz, the area of the truncated problems of moments saw interesting development in various other directions, that we do not attempt to cover. A few recent works [5] - 9] should be mentioned in this sense, see also [3, [12, [15], [16].

I express thanks to professor Raul Curto and professor Lawrence Fialkow for drawing the analogue of the Riesz-Haviland theorem in the truncated case [6] to my attention.

\section{Main results}

Definitions Let $T \neq \emptyset$ be a closed subset of $\mathbb{R}^{n}$, such that for any $t \in T$ and $\varepsilon>0$ the Lebesgue measure of the set $\{x \in T:\|x-t\|<\varepsilon\}$ is $>0$. This always holds a.e. (in the density points of $T[7.6,[17]]$ ), but we require it in every point $t$. We call such a $T$ regular. Here \|\| is the usual Euclidian norm. For any multiindex $i \in \mathbb{Z}_{+}^{n}$, set $\sigma_{i}=\left\{j \in \mathbb{Z}_{+}^{n}: j_{k}=\right.$ either 0 or $\left.i_{k}, 1 \leq k \leq n\right\}$. Let $I \subset \mathbb{Z}_{+}^{n}$ be finite, $I \neq \emptyset$ such that $\sigma_{i} \subset I$ for all $i \in I$. We call such an $I$ regular, too. Let $g=\left(g_{i}\right)_{i \in I}$ be a set of real numbers with $g_{0}=1$. We call a convex cone $C$ in a real linear space $F$ acute if $C \cap(-C)=\{0\}$. Given also a linear functional $f: F \rightarrow \mathbb{R}$, we write $f>0$ if $f c>0$ for all $c \in C \backslash\{0\}$.

Lemma 2 and its Corollary 3 follow from various well known arguments (Hahn-Banach, Krein, Mazur, Choquet [4, 10, 17]) on the extension of positive functionals. We found it easier to gather them in a short proof.

Lemma 2 Let $C \subset F$ be an acute closed convex cone in a finite dimensional linear space $F$. Let $L \subset F$ be a linear subspace of codimension 1, and $\phi$ a linear functional on $L$ such that $\phi l>0$ for all $l \in L \cap C$ with $l \neq 0$. Then there is an extension $\Phi$ of $\phi$ to $F$ such that $\Phi x>0$ for all $x \in C, x \neq 0$.

Proof. The sum $Y+\mathbb{R} v$ of a closed convex cone $Y \subset F$ and a 1-dimensional subspace $\mathbb{R} v$ is closed. Indeed, if both $\pm v \in Y, \mathbb{R} v \subset Y$ and so $Y+\mathbb{R} v=$ $Y$; if not, we may suppose $v \notin Y$ by replacing $v$ by $-v$ if necessary. Let 
$x=\lim _{k \rightarrow \infty}\left(y_{k}+\lambda_{k} v\right)$ where $y_{k} \in Y$. If $\left(\lambda_{k}\right)_{k}$ is bounded, by compactness we obtain a number $\lambda$ and vector $y \in Y$ such that $x=y+\lambda v$. If it is not, we can assume either $\lim _{k} \lambda_{k}=\infty$, or $\lim _{k} \lambda_{k}=-\infty$. From $y_{k}+\lambda_{k} v \rightarrow x$ we derive $\frac{1}{\lambda_{k}} y_{k}+v \rightarrow 0$. The case $\lambda_{k} \rightarrow-\infty$ is impossible since it leads to $v=\lim _{k} \frac{1}{-\lambda_{k}} y_{k} \in Y$. If $\lambda_{k} \rightarrow \infty$, we obtain $-v=\lim _{k} \frac{1}{\lambda_{k}} y_{k} \in Y$, and so the distance $d(x, Y)$ from $x$ to $Y$ satisfies $d(x, Y)=\lim _{k} d\left(y_{k}-\lambda_{k}(-v), Y\right)=$ $\lim _{k} d\left(y_{k}, Y\right)=0$ whence $x \in Y$. In particular we get (inductively) that the convex cone $K:=C+\operatorname{ker} \phi$ is closed.

To find $\Phi$, we may suppose $C \neq\{0\}$. If $\phi=0, L \cap C=\{0\}$. Let then $f$ be a linear functional on $F$ with $\operatorname{ker} f=L$. Since $C$ is acute, $C \backslash\{0\}$ is segmentwise connected. Hence the set $I:=f(C \backslash\{0\})$ is connected, and so, an interval, that cannot contain 0 for: $f c=0, c \in C \Rightarrow c \in L \cap C=\{0\}$. Then either $I \subset(0, \infty)$, in which case we let $\Phi=f$, or $I \subset(-\infty, 0)$ in which case $\Phi:=-f$. Then $\Phi c \geq 0$ for all $c \in C$, with strict inequality if $c \neq 0$.

If $\phi \neq 0$, let $F^{\prime}=F / \operatorname{ker} \phi$ and $\lambda: F \rightarrow F^{\prime}$ be the factorization map. Then $C^{\prime}:=\lambda(C), L^{\prime}:=\lambda(L)$ and the map $\phi^{\prime}$ induced by $\phi$ on $L^{\prime}$ satisfy the hypotheses as well. Indeed, $K=C+\operatorname{ker} \lambda$ is closed, $\lambda$ open and $\lambda(F \backslash K)=F^{\prime} \backslash C^{\prime}$ whence $C^{\prime}$ is closed. Also $\phi>0, C$ acute $\neq\{0\} \Rightarrow \phi^{\prime}>0, C^{\prime}$ acute $\neq\{0\}$. Since $\operatorname{dim} F^{\prime}=2$, we easily get the existence of an extension $\Phi^{\prime}>0$ of $\phi^{\prime}$ to $F^{\prime}$, that will provide $\Phi:=\Phi^{\prime} \circ \lambda$ : note that $\operatorname{dim} L^{\prime}=1$ and $\left.\phi^{\prime}\right|_{L^{\prime}}$ is injective, and so increasing along a direction of $L^{\prime}$ given by a vector $\bar{e} \in \mathbb{R}^{2}$ (a drawing would help). Then $L^{\prime}=$ $\{r \bar{e}\}_{r \in \mathbb{R}}$ and $\phi^{\prime} \bar{e}>\phi^{\prime} 0=0$. The (closed) convex cone $\mathcal{C}:=\operatorname{co}\left(C^{\prime}, \bar{e}\right)$ generated by $C^{\prime}$ and $\bar{e}$ is acute, for otherwise $\left.\phi^{\prime}\right|_{L^{\prime}}$ would decrease and be $<0$ along the half-line $\{r \bar{e}\}_{r<0}$ opposite to $\bar{e}$ and contained into $C^{\prime}$, which is impossible since $\phi^{\prime}>0$ on $L^{\prime} \cap C^{\prime} \backslash\{0\}$. Since $\mathcal{C}$ is acute, there is an extension $\Phi^{\prime}>0$ of $\phi^{\prime}$, whose kernel is a supporting line for $\mathcal{C}$ in 0 only: fix $\bar{f} \in \mathbb{R}^{2}$ such that $(\mathbb{R} \bar{f}) \cap \mathcal{C}=\{0\}$, then for any $v=r \bar{e}+s \bar{f}$ with $r, s$ real, set $\Phi^{\prime} v=r \phi^{\prime} \bar{e}$. If $v \in C^{\prime} \backslash\{0\}, r>0$ and so $\Phi^{\prime} v=r \phi^{\prime} \bar{e}>0$.

Corollary 3 Let $F$ be a finite dimensional linear space and $K \subset F$ an acute closed convex cone. Let $f_{0}: L \rightarrow \mathbb{R}$ be a linear functional on a linear subspace $L$ of $F$ such that $f_{0} x>0$ for every $x \in L \cap K$ with $x \neq 0$. Then there is a linear extension $f$ of $f_{0}$ to $F$ such that $f x>0$ for every $x \in K, x \neq 0$.

For any $\sigma$-finite measure $\mu \geq 0$ on $T$ and $1 \leq p \leq \infty$ the symbols $L^{p}(T, \mu)$, $L_{+}^{p}(T, \mu)$ have the usual meaning. Lemma 4 is an extension of [Theorem 2.9, [2]]. We give its original proof adapted to our slightly different context.

Lemma 4 (see 2]) Let $T \subset \mathbb{R}^{n}$ be closed with positive Lebesgue measure, finite or not. Let $\rho: T \rightarrow(0, \infty)$ be locally integrable, and $\mu=\rho d t$ be the measure on $T$ with density $\rho$. Let $I \subset \mathbb{Z}_{+}^{n}$ be finite. Let $f \geq 0$ a.e. on $T$ be measurable, $f \not \equiv 0$ a.e., such that $\int_{T}\left|t^{i}\right| f(t) d \mu(t)<\infty(i \in I)$. There is an $r>0$ such that for any $\beta=\left(\beta_{i}\right)_{i \in I}$ with $\|\beta\|<r$, there exists a $g=g_{\beta} \in L^{\infty}(T, \mu), g>0$ a.e. with the properties $\int_{T}\left|t^{i}\right| g(t) d \mu(t)<\infty$,

$$
\int_{T} t^{i} g(t) d \mu(t)=\int_{T} t^{i} f(t) d \mu(t)+\beta_{i} \quad(i \in I) .
$$


Proof. Set $T_{l}=\{t \in T: f(t) \geq 1 / l,\|t\| \leq l\}$. Using $\{t \in T: f(t)>0\}=$ $\cup_{l \in \mathbb{N}} T_{l}$ we find $\delta>0$ and $T_{*} \subset T$ bounded with $0<\mu\left(T_{*}\right)<\infty$ such that $f(t) \geq \delta$ a.e. on $T_{*}$. The map $A: L^{\infty}\left(T_{*}\right) \rightarrow \mathbb{R}^{N}(N=\operatorname{card} I)$ given by $A u=\left(\int_{T_{*}} u(t) t^{i} d \mu(t)\right)_{i \in I}$ is surjective, for if there is a vector $\lambda=\left(\lambda_{i}\right)_{i} \neq 0$ orthogonal to its range, $\sum_{i} \lambda_{i} \int_{T_{\star}} u t^{i} d \mu=0 \forall i$, u whence $\sum_{i} \lambda_{i} t^{i}=0$ a.e. on $T_{*}$, that is impossible because the set of zeroes of a polynomial $\not \equiv 0$ has measure zero. Then $A$ is open. Hence 0 is in the interior int $C$ of the set $C=\left\{A u: u \in L^{\infty}\left(T_{*}, d t\right),\|u\|<\delta / 4\right\}$. Fix $r>0$ such that the ball of center 0 and radius $r$ is contained in $C$. Define $f_{k}$ on $T$ by $f_{k}(t)=\min (f(t), k)+$ $\frac{1}{k} e^{-\|t\|} / \rho(t)$. Then $0 \leq\left|t^{i}\right| f_{k} \leq\left|t^{i}\right| f+\left|t^{i}\right| e^{-\|t\|} / \rho(t) \in L^{1}(T, \mu)$ and $f_{k} \rightarrow f$ a.e. as $k \rightarrow \infty$. The vector $\left(\int_{T}\left(f_{k}-f\right) t^{i} d \mu\right)_{i \in I} \rightarrow 0$ in $\mathbb{R}^{N}$ as $k \rightarrow \infty$, by Lebesgue's theorem of dominated convergence. Since $0 \in \operatorname{int} C$, for large $k$ we have $\left(\int_{T}\left(f_{k}-f\right) t^{i} d \mu\right)_{i} \in C$. Then $\int_{T}\left(f_{k}-f\right) t^{i} d \mu=\int_{T} u t^{i} d \mu$ for some $u \in L^{\infty}(T)$ with $\|u\|<\delta / 4$ and $u=0$ outside $T_{*}$. Also, if $\|\beta\|<r, \beta \in C$ and so there is a $v \in L^{\infty}(T), v=0$ outside $T_{*}$, with $\|v\|<\delta / 4$ such that $\int_{T} v t^{i} d \mu=\beta_{i}$. Set $g=f_{k}-u+v$ for a sufficiently large fixed $k(\geq \delta)$. Hence $\int_{T} g t^{i} d \mu=\int_{T}\left(f_{k}-u+v\right) t^{i} d \mu=\int_{T}\left(f_{k}-u\right) t^{i} d \mu+\int_{T} v t^{i} d \mu=\int_{T} f t^{i} d \mu+\beta_{i}$. Since $\rho$ is locally integrable and $T_{*}$ contained in a ball, on which $u, v$ and all $t^{i}$ are bounded, the functions $t^{i} u, t^{i} v \in L^{1}(T, \mu)$. Hence $\left|t^{i}\right| g=\left|t^{i}\right|\left(f_{k}-u+v\right)$ is in $L^{1}(T, \mu)$. Moreover $f_{k}, u, v$ (and hence, $g$ ) are in $L^{\infty}(T)$. On $T_{*}, f \geq \delta$ and $|u|,|v| \leq \delta / 2$, whence $g=f_{k}-u+v \geq \min (f, k)-u+v \geq \delta-u+v \geq \delta / 2$. Outside $T_{*}, g=f_{k} \geq \frac{1}{k} e^{-\|t\|} / \rho(t)>0$. Then $g>0$ a.e.

Notation Given any closed subset $T \subset \mathbb{R}^{n}$ and finite subset $I \subset \mathbb{Z}_{+}^{n}$, set $\Gamma_{T I}=\left\{\gamma=\left(\gamma_{i}\right)_{i \in I}: \exists\right.$ Borel measures $\nu \geq 0$ on $T$ with $\left.\int_{T} t^{i} d \nu(t)=\gamma_{i}, i \in I\right\}$ and $G_{T I}=\left\{g=\left(g_{i}\right)_{i \in I} \neq 0: \exists f \in L_{+}^{1}(T, d t)\right.$ such that $\left.\int_{T} t^{i} f(t) d t=g_{i}, i \in I\right\}$, where $\left|t^{i}\right|$ are implicitely supposed to be integrable.

Lemma 5 Let $T \subset \mathbb{R}^{n}$ be a closed regular set and $I \subset \mathbb{Z}_{+}^{n}$ a finite regular set. Then $G_{T I}$ is dense in $\Gamma_{T I}$.

Proof. Let $\gamma \in \Gamma_{T I}$. There is a measure $\nu \geq 0$ on $T$ such that

$$
\int_{T} y^{i} d \nu(y)=\gamma_{i} \quad(i \in I)
$$

in particular $\nu(T)<\infty$ since $0 \in I$. For any $\varepsilon \in(0,1)$ let $h_{\varepsilon}$ be the characteristic function of the ball $b_{\varepsilon}$ of center 0 and radius $\varepsilon$ in $\mathbb{R}^{n}, h_{\varepsilon}=1$ on $b_{\varepsilon}$ and $h_{\varepsilon}=0$ outside $b_{\varepsilon}$. For any $y \in T$, let $v_{\varepsilon}(y)$ be the $n$-dimensional volume of the set $\{x \in T:\|x-y\|<\varepsilon\}$. Then $v_{\varepsilon}=\left.\left(h_{\varepsilon} * h_{T}\right)\right|_{T}$ is the convolution of $h_{\varepsilon}$ with the characteristic function $h_{T}$ of $T$. Hence the map $y \mapsto v_{\varepsilon}(y)$ is measurable. All $v_{\varepsilon}(y)>0$ since $T$ is regular. For $t \in T$, set $\nu_{\varepsilon}(t)=\int_{T} \frac{1}{v_{\varepsilon}(y)} h_{\varepsilon}(t-y) d \nu(y)$. By the Tonelli and Fubini theorems, $\nu_{\varepsilon} \in L_{+}^{1}(T, d t)$ has finite moments of orders $i \in I$ on $T$, that we compute by

$$
\int_{T} t^{i} \nu_{\varepsilon}(t) d t=\int_{T} \frac{1}{v_{\varepsilon}(y)} \int_{T} t^{i} h_{\varepsilon}(t-y) d t d \nu(y)=\int_{T} \psi_{\varepsilon i}(y) d \nu(y)
$$


where $\psi_{\varepsilon i}(y)=\frac{1}{v_{\varepsilon}(y)} \int_{T} t^{i} h_{\varepsilon}(t-y) d t$. By the change of variables $t-y=w$,

$\psi_{\varepsilon i}(y)=\frac{1}{v_{\varepsilon}(y)} \int_{\|w\|<\varepsilon, w \in T-y}(y+w)^{i} d w=y^{i} \frac{1}{v_{\varepsilon}(y)} \int_{\|w\|<\varepsilon, w \in T-y} d w+E_{i}(\varepsilon, y)$

where $E_{i}(\varepsilon, y)$ is a linear combination with binomial coefficients $c_{i j}$,

$$
E_{i}(\varepsilon, y)=\sum_{0 \leq j \leq i, j \neq i} c_{i j} y^{j} \frac{1}{v_{\varepsilon}(y)} \int_{\|w\|<\varepsilon, w \in T-y} w^{i-j} d w
$$

and the order on $\mathbb{Z}_{+}^{n}$ is given as usual by $j \leq i \Leftrightarrow j_{k} \leq i_{k}$ for $1 \leq k \leq n$. The set $\{\|w\|<\varepsilon, w \in T-y\}$ is taken by the translation $w \mapsto w+y$ into the set $\{x \in T:\|x-y\|<\varepsilon\}$ the Lebesgue measure of which is $v_{\varepsilon}(y)$. Hence

$$
\frac{1}{v_{\varepsilon}(y)} \int_{\|w\|<\varepsilon, w \in T-y} d w=1
$$

Then

$$
\psi_{\varepsilon i}(y)=y^{i}+E_{i}(\varepsilon, y) .
$$

Since $\|w\|<\varepsilon<1$ and $|i-j| \geq 1$ for all $j$ in (2), $\left|w^{i-j}\right| \leq\|w\||i-j| \leq\|w\|<\varepsilon$. Hence by (2) and (4), we obtain the estimate

$$
\left|E_{i}(\varepsilon, y)\right| \leq \varepsilon \sum_{0 \leq j \leq i} c_{i j}\left|y^{j}\right| .
$$

Since $I$ is regular, $j \leq i \Rightarrow\left|y_{k}^{j_{k}}\right| \leq\left|y_{k}^{i_{k}}\right|+1 \Rightarrow\left|y^{j}\right| \leq \prod_{k=1}^{n}\left(\left|y_{k}^{i_{k}}\right|+1\right)=$ $\sum_{\iota \in \sigma_{i}}\left|y^{\iota}\right| \leq \sum_{\iota \in I}\left|y^{\iota}\right|$ and so we can integrate in (5), (6) with respect to $\nu$ on $T$. By (2) and (11), this gives $\lim _{\varepsilon \rightarrow 0} \int_{T} t^{i} \nu_{\varepsilon}(t) d t=\gamma_{i}$ for all $i \in I$. Set $\tilde{\gamma}_{\varepsilon}=\left(\int_{T} t^{i}\left(\nu_{\varepsilon}(t)+\varepsilon e^{-\|t\|}\right) d t\right)_{i \in I}$. Then $\tilde{\gamma}_{\varepsilon} \in G_{T I}$ and $\lim _{\varepsilon \rightarrow 0} \tilde{\gamma}_{\varepsilon}=\gamma$.

Theorem 6 Let $T \subset \mathbb{R}^{n}$ be a closed regular set, and $I \subset \mathbb{Z}_{+}^{n}$ a finite regular set. Then $G_{T I}$ is the dense interior of $\Gamma_{T I}$.

Proof. By Lemma 4 for $\rho \equiv 1, G_{T I}$ is open, and so contained in the interior of $\Gamma_{T I}$ (the regularity of $T, I$ is not required here). Let $\gamma$ be in the interior of $\Gamma_{T I}$. There is an $r>0$ such that the ball $B$ of center $\gamma$ and radius $r$ is contained in $\Gamma_{T I}$ (a drawing will be helpful). By Lemma 5 there is a $\tilde{\gamma} \in G_{T I} \cap B$. By Lemma 4 applied to a representing density $f$ of $\tilde{\gamma}$, there is an $r_{0}^{\prime}>0$ such that all balls $B\left(\tilde{\gamma}, r^{\prime}\right)$ of center $\tilde{\gamma}$ and radii $r^{\prime} \in\left(0, r_{0}^{\prime}\right]$ satisfy $B\left(\tilde{\gamma}, r^{\prime}\right) \subset G_{T I}$. We can fix an $r^{\prime}$ sufficiently small so that $B\left(\tilde{\gamma}, r^{\prime}\right) \subset B$. Let $\gamma^{\prime}$ be the unique point such that $\gamma=\frac{1}{2}\left(\tilde{\gamma}+\gamma^{\prime}\right)$. Since $B\left(\tilde{\gamma}, r^{\prime}\right) \subset B$, then $B\left(\gamma^{\prime}, r^{\prime}\right) \subset B$; a quick argument to this aim is that $B$ is symmetric with respect to its center $\gamma$ and $B\left(\gamma^{\prime}, r^{\prime}\right)$, $B\left(\tilde{\gamma}, r^{\prime}\right)$ are symmetric to each other over $\gamma$. In particular $\gamma^{\prime} \in B \subset \Gamma_{T I}$. By Lemma 5 there is a $\tilde{\gamma}^{\prime} \in G_{T I} \cap B\left(\gamma^{\prime}, r^{\prime}\right)$. Since $\tilde{\gamma}^{\prime} \in B\left(\gamma^{\prime}, r^{\prime}\right)$, the point $v$ such that $\gamma=\frac{1}{2}\left(\tilde{\gamma}^{\prime}+v\right)$ must be in $B\left(\tilde{\gamma}, r^{\prime}\right)\left(\subset G_{T I}\right)$. Hence $v \in G_{T I}$. Since $G_{T I}$ is convex, $\gamma=\frac{1}{2}\left(\tilde{\gamma}^{\prime}+v\right)$ and both $\tilde{\gamma}^{\prime}, v \in G_{T I}$, then $\gamma \in G_{T I}$. 
By the previous results, the following completion (Theorem 7) can be made to the Riesz-Haviland theorem [13] and its truncated version [6].

Condition (b) from below is equivalent to the existence of a constant $c>0$ such that $\varphi_{g} p \geq c\|p\|$ for every $p \in P_{I}$ with $p(t) \geq 0$ for all $t \in T$, where \|\| is any norm on $P_{I}$ : use the compactness of $\left\{p \in P_{I}: p \geq_{T} 0,\|p\|=1\right\}$ and write $\varphi_{g}(p /\|p\|)>0$ for such $p \in P_{I} \backslash\{0\}$.

Theorem 7 Let $T \subset \mathbb{R}^{n}$ be a closed regular set. Let $I \subset \mathbb{Z}_{+}^{n}$ be a finite regular set. Let $g=\left(g_{i}\right)_{i \in I}$ be a set of numbers with $g_{0}=1$. The following statements are equivalent:

(a) There exist functions $f \in L_{+}^{1}(T, d t)$ such that $\int_{T}\left|t^{i}\right| f(t) d t<\infty$ and

$$
\int_{T} t^{i} f(t) d t=g_{i} \quad \text { for all } i \in I
$$

(b) The Riesz functional $\varphi_{g}$ defined on the linear span $P_{I} \subset \mathbb{R}\left[X_{1}, \ldots, X_{n}\right]$ of the monomials $X^{i}, i \in I$ by

$$
\varphi_{g} \sum_{i \in I} c_{i} X^{i}=\sum_{i \in I} g_{i} c_{i}
$$

satisfies $\varphi_{g} p>0$ for every $p \in P_{I} \backslash\{0\}$ such that $p(t) \geq 0$ for all $t \in T$.

Proof. $\quad(\mathrm{a}) \Rightarrow$ (b) From $\varphi_{g} p=\int_{T} p f d t$ we obtain as usual that $\varphi_{g} p \geq 0$ for every polynomial $p$ such that $p(t) \geq 0$ for all $t \in T$. If moreover $p \neq 0$ in $\mathbb{R}\left[X_{1}, \ldots, X_{n}\right]$, the set $Z=\left\{t \in \mathbb{R}^{n}: p(t)=0\right\}$ of the zeroes of $p$ is an algebraic variety, (empty or) of dimension $\leq n-1$, and so has null Lebesgue measure. Then $\varphi_{g} p>0$, for the equality $\int_{T} p f d t=\int_{T \backslash Z} p f d t=0$ with $p>0$ on $T \backslash Z$ would compel $f=0_{L^{1}(T, d t)}$ that is impossible since $\int_{T} f d t=g_{0}=1$.

(b) $\Rightarrow$ (a) Endow $P_{I}$ with a norm and its dual $P_{I}^{*}$ with the dual norm. Let $C$ denote the convex cone of all $p \in \mathbb{R}\left[X_{1}, \ldots, X_{n}\right]$ such that $p(t) \geq 0$ for all $t \in T$. Since the Lebesgue measure of $T$ is $>0, C$ is acute. There is a constant $c=c_{g}>0$ such that $\varphi_{g} p \geq c\|p\|$ for every $p \in P_{I} \cap C$, see the comment just before the Theorem. Since the map $\gamma \mapsto \varphi_{\gamma} \in P_{I}^{*}$ is linear, there is a constant $c^{\prime}>0$ such that $\left\|\varphi_{\gamma}\right\| \leq c^{\prime}\|\gamma\|$ for all $\gamma$. Then for every $\tilde{g}$ in the ball $B$ of center $g$ and radius $r=c / 2 c^{\prime}$, we have $\varphi_{\tilde{g}} p \geq(c / 2)\|p\|$ for all $p \in P_{I} \cap C$. Indeed, $\varphi_{\tilde{g}} p=\varphi_{g} p+\varphi_{\tilde{g}-g} p \geq c\|p\|-\left|\varphi_{\tilde{g}-g} p\right| \geq c\|p\|-c^{\prime}\|\tilde{g}-g\|\|p\| \geq(c / 2)\|p\|$. Hence condition (b), briefly $\varphi_{\tilde{g}}>0$, holds as well for all $\tilde{g}$ in the neighborhood $B$ of $g$. Write $\mathbb{Z}_{+}^{n}=\cup_{l \in \mathbb{N}} I_{l}$ as an increasing union of finite subsets $I_{l}$ such that $I_{1}=I$. Let $P_{l}$ be the linear span of the monomials $X^{i}$ with $i \in I_{l}$. Thus $P_{1}=P_{I}$. Let $\tilde{g}=\left(\tilde{g}_{i}\right)_{i \in I}$ be an arbitrary point in $B$, that for the moment we fix. Since $\varphi_{\tilde{g}}>0$, by applying successively Corollary 3 for $F=P_{l+1}, K=C \cap P_{l+1}$ and $L=P_{l}$ with $l \geq 1$ we obtain, starting from $\psi_{1}:=\varphi_{\tilde{g}}: P_{1} \rightarrow \mathbb{R}$, a sequence of linear functionals $\psi_{l \tilde{g}}: P_{l} \rightarrow \mathbb{R}$ such that $\left.\psi_{l+1 \tilde{g}}\right|_{P_{l}}=\psi_{l \tilde{g}}$ for all $l \geq 1$ and $\psi_{l \tilde{g}} p>0$ for all $p \in P_{l} \cap C \backslash\{0\}$. Then we have a linear functional $\psi_{\tilde{g}}: \mathbb{R}\left[X_{1}, \ldots, X_{n}\right] \rightarrow \mathbb{R}$, extending $\varphi_{\tilde{g}}$, determined by $\left.\psi_{\tilde{g}}\right|_{P_{l}}=\psi_{l \tilde{g}}$ for all $l \geq 1$. For any polynomial $p \in C$, $p \not \equiv 0$ there is an $l \geq 1$ such that $p \in P_{l}$, and so $\psi_{\tilde{g}} p=\psi_{l \tilde{g}} p>0$. Hence $\psi_{\tilde{g}} p \geq 0$ 
for every polynomial $p$ such that $p(t) \geq 0$ for all $t \in T$. By the Riesz-Haviland's Theorem 1 there is a measure $\nu_{\tilde{g}} \geq 0$ on $T$, with finite moments of any order, such that $\psi_{\tilde{g}} p=\int_{T} p d \nu_{\tilde{g}}$ for every polynomial $p$. In particular for $p:=X^{i}$ with $i \in I$, we obtain $\tilde{g}_{i}=\varphi_{\tilde{g}} X^{i}=\psi_{\tilde{g}} X^{i}=\int_{T} t^{i} d \nu_{\tilde{g}}(t)$. Thus $\tilde{g}_{i}(i \in I)$ are the moments of a measure $\nu_{\tilde{g}}$ on $T$, that is, $\tilde{g} \in \Gamma_{T I}$. Since $\tilde{g}$ was arbitrary in a neighborhood of $g$, it follows that $g$ is in the interior of $\Gamma_{T I}$. Then by Theorem 6. $g \in G_{T I}$.

Acknowledgement The work was supported by the grants IAA100190903 GAAV and 201/09/0473 GACR, RVO: 67985840.

\section{References}

[1] N.I. Akhiezer, The classical moment problem, Hafner Publ. Co., New York, 1965.

[2] J.M. Borwein; A.S. Lewis, Duality relationships for entropy-like minimization problems, SIAM J. Cont. Optimization 29:2(1991), 325-338.

[3] G. Cassier, Problème des moments sur un compact de $\mathbb{R}^{n}$ et décomposition de polinômes à plusieurs variables, J. Funct. Analysis, 58(1984), 254-266.

[4] G. Choquet, Le problème des moments, Séminaire d'initiation à l'analyse. Institut Henri Poincaré, Paris, $1^{\text {re }}$ année, 1962, 10 pages.

[5] D. Cichoń; J. Stochel; F.H. Szafraniec, Riesz-Haviland criterion for incomplete data, J. Math. Anal. Appl. 380:1(2011), 94-104.

[6] R.E. Curto; L.A. Fialkow, An analogue of the Riesz-Haviland theorem for the truncated moment problem, J. Funct. Anal. 255:10(2008), 2709-2731.

[7] R.E. Curto; L.A. Fialkow, Truncated $K$-moment problems in several variables, J. Operator Theory 54(2005), 189-226.

[8] R.E. Curto; L.A. Fialkow, Solution of the truncated complex moment problem for flat data, Memoirs of the A.M.S. 1996.

[9] R.E. Curto; L.A. Fialkow; H.M. Moeller, The extremal truncated moment problem, Integral Equations Operator Theory 60:2(2008), 177-200.

[10] N. Dunford; J.T. Schwartz, Linear operators. Part I: General theory, Interscience Publications, New York, 1953.

[11] L.A. Fialkow; J. Nie, Positivity of Riesz functionals and solutions of quadratic and quartic moment problems, J. Funct. Anal. 258:1(2010), 328356.

[12] B. Fuglede, The multidimensional moment problem, Exp. Math. 1(1983), 47-65. 
[13] E.K. Haviland, On the momentum problem for distributions in more than one dimension I, Amer. J. Math. 57(1935), 562-568; II, 58(1936), 164-168.

[14] M. Junk, Maximum entropy for reduced moment problems, Math. Models Methods Appl. Sci. 10:7(2000), 1001-1025.

[15] A. Prestel; C.N. Delzell, Positive polynomials. From Hilbert's 17th problem to real algebra. Springer Monographs in Mathematics. Springer, Berlin, 2001.

[16] M. Putinar; F.-H. Vasilescu, Solving moment problems by dimensional extension, Ann. of Math. (2) 149:3(1999), 1097-1107.

[17] W. Rudin, Real and complex analysis, 3rd edition 1987, 1974, 1966 by McGraw-Hill, Inc.

[18] J. Shohat; J. Tamarkin, The problem of moments, Math. Surveys I, Amer. Math. Soc., Providence, RI, 1943.

Institute of Mathematics, AS CR

Zitna 25

11567 Prague 1

Czech Republic

ambrozie@math.cas.cz

and: Institute of Mathematics "Simion Stoilow" - Romanian Academy,

PO Box 1-764, 014700 Bucharest, Romania 